\title{
Collective dynamics in dispersions of anisometric pigment particles $^{\text {th }}$
}

\author{
Kathrin May ${ }^{\mathrm{a}}$, Ralf Stannarius ${ }^{\mathrm{a}}$, Kyongok Kang ${ }^{\mathrm{b}}$, Pavan Challa Kumar ${ }^{\mathrm{c}}$, Samuel Sprunt ${ }^{\mathrm{c}}$, Antal \\ Jáklid $^{\mathrm{d}}$, Susanne Klein ${ }^{\mathrm{e}}$, Alexey Eremin ${ }^{\mathrm{a}}$ \\ ${ }^{a}$ Otto von Guericke University, Institute for Experimental Physics, Dept. for Nonlinear Phenomena, 39106 Magdeburg, \\ Germany \\ ${ }^{b}$ Research Center in Jülich, ICS-3 (Soft Condensed Matter), Jülich, 52425, Germany \\ ${ }^{c}$ Department of Physics, Kent State University, Kent, OH, 44242, USA \\ ${ }^{d}$ Liquid Crystal Institute, Kent State University, Kent, OH 44242, USA \\ ${ }^{e}$ HP Laboratories, Long Down Avenue, Stoke Gifford, Bristol BS34 8QZ, UK
}

\begin{abstract}
Dynamics of suspensions of solid rodlike pigment particles in a non-polar solvent were studied in a concentration range from the isotropic up to orientationally ordered nematic-like phase. Using dynamic light scattering and gradient recovery measurements, we studied the rotational and translational diffusion coefficients. We demonstrate that the translational diffusion coefficient in this system is increasing with increasing concentration of the pigment particles in the vicinity of the transition into an ordered phase. This unexpected behaviour can be attributed to the collective interactions between the particles and the alignment effects.
\end{abstract}

Keywords: anisometric colloids, dynamics, diffusion, pigments

\section{Introduction}

Colloidal suspensions have been an active area of scientific research for almost a century now. Such suspensions mimic the behaviour of molecular materials where the colloidal particles play a role of "molecules". The interactions between colloidal particles can be fine-tuned by variation of the dispersant composition and/or modification of the particle surfaces $[1,2]$. As a result, such dispersions exhibit a variety of self-assembly phenomena including gas-liquid and liquid-solid or glass transitions. Another type of self-assembly observed in the molecular systems is based on the spontaneous breaking of rotational symmetry and development of an orientationally ordered fluid state of matter - liquid crystals [3]. To achieve this kind of symmetry breaking instability in colloidal dispersions, their constituting particles require an anisometric (shape-anisotropic) form. A variety of systems consisting of rigid and semi-flexible particles have been found to show liquid crystalline order and phase behaviour typical for lyotropic liquid crystals. These include tobacco mosaic virus (TMV) [4, 5, 6], fd-virus [7, 8, 9, 10], clay particles, gibbsite and magnetic particles such as goethite and hematite spindles $[11,12]$. The complexity

\footnotetext{
This paper is dedicated to the memory Yuriy Reznikov, to whom we owe pioneering contributions to the study of anisotropic suspensions.
} 
of the mesophase behaviour of colloidal liquid crystals is comparable with that of molecular systems where nematic, smectic and hexatic phases occur. These systems are responsive to external electric and magnetic fields. Electro- and magneto-responsive suspensions have found broad applications in the design of engine clutches and coatings for data storage device [13,14]. Anther important colloidal system often used in everyday life is ink or paint. Inks consist of solid pigment particles, often with anisometric shapes, dispersed in various dispersants. An important feature of anisometric pigment particles is dichroism [15]: optical absorption of a particle depends on the light polarisation. Such pigment particles can be easily aligned in an electric field, resulting in a birefringent, dichroic state $[16,17]$. This makes such pigments good candidates for the development of dichroic displays. Colloidal suspensions of form-anisometric particles exhibit remarkable dynamic behaviour. Orientational fluctuations of the particles become strongly correlated, and the translational diffusion becomes anisotropic. In case of particles in a smectic matrix, self-diffusion occurs as a hopping-type process across the layers [18]. Long charged fd-viruses exhibit even a structural arrest resulting in a glass transition at a low ionic strength $[19,20,21]$.

In our previous study [17], we showed that suspensions of the pigment particles used here form an orientationally ordered (nematic) phase at volume fractions as low as 17 vol\% and above. At moderate volume fractions, 12-17 vol\%, the suspensions exhibit a globally isotropic state with enhanced orientational fluctuations. This is manifested in a strong optical response (birefringence) to an externally applied electric field. Recently, we demonstrated that non-polar suspensions of rod-shaped pigment particles exhibit, in addition to mesomorphic behaviour, a strong electro-optical response and a rich morphology of spontaneously formed patterns in AC fields $[17,22,23]$. These suspensions were proposed as possible candidates for display applications, particularly with such particles dispersed in a thermotropic liquid crystalline host [16].

One of the consequences of the non-spherical particle shapes is the unconventional dynamics on the micro-scale, which is reflected in macroscopic phenomena like spontaneous selforganisation and pattern formation, unusual flow characteristics and anisotropic physical properties. Collective dynamics and alignment effects compete with effects of particle densities in a complex way. This paper describes the dynamics of pigment particles, and their characterisation using the optical imaging techniques and dynamic light scattering. In the investigated system, a complex concentration dependence of diffusion coefficients evidences the dramatic consequences of the above mentioned competition.

\section{System and Experimental Methods}

The fluorescent dye used here is C.I. Pigment Red 176, a blue shade benzimidazolone pigment. Rod-like particles labeled with this dye are commercially available under the name Novoperm Carmine HF3C (Clariant, Frankfurt am Main, Germany), they are used as received. The rods have a mean length $L$ of $230 \mathrm{~nm} \pm 70 \mathrm{~nm}$ and a mean diameter $D$ of $46 \mathrm{~nm} \pm 20 \mathrm{~nm}$ (see Fig. 1b) $[24]$

The pigment crystallites were suspended in the non-polar solvent dodecane (Sigma-Aldrich, Hamburg, Germany, used as received) using the commercially available polymeric dispersant Solsperse 11200 (Lubrizol, Brussels, Belgium, used as received). The stabiliser is a dispersant comprising an amine carrying a poly(carbonylalkyleneoxy) chain (PCAO chain) in which the alkylene groups contain up to 8 carbon atoms, or an acid-salt thereof, as disclosed in [25]. The stabiliser partially adsorbs on the particle surface. A significant fraction of the stabiliser, however remains in the solution [26]. The concentration of the stabiliser was chosen to minimise 
a)

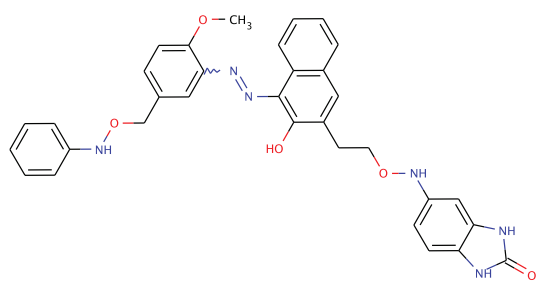

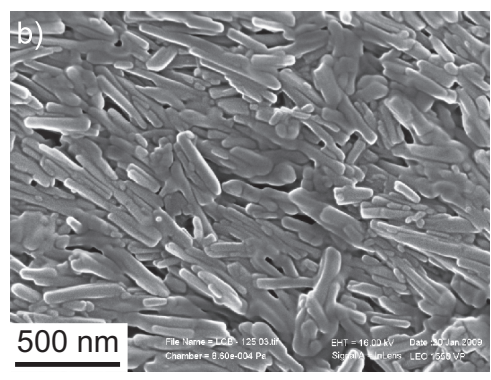

Figure 1: a) Chemical formula of a C.I. Pigment Red 176. b) Scanning electron microscopy (SEM) image of the pigment particles.

the hydrodynamic radius of the particles. First, suspensions with pigment concentrations of 20 $\mathrm{wt} \%$ and above were prepared by milling. Thereafter, $40 \mathrm{wt} \%$ of dispersant per weight of the pigment were added and dissolved. After the addition of the pigment, the mixture was milled in a planetary mill (Fritsch Pulverisette 7 premium line), using 0.3-mm Yttria-stabilised Zirconia beads in Zirconia-lined pots for a total of 60 minutes at $500 \mathrm{rpm}$. Appropriate cooling cycles were kept in order to prevent the temperature inside the pots to rise above $60{ }^{\circ} \mathrm{C}$. Concentrations below $20 \mathrm{wt} \%$ were then made by stepwise dilution of the base suspension. In order to test the stability, the samples were centrifuged at $10000 \mathrm{rpm}$ for 60 minutes. None of the concentrations showed a separation in particle-rich and particle-poor zones. Even after 12 months, samples left untouched did not show any phase separation or aggregation.

Two methods are used to study the dynamics of the resulting dispersions: a depletion recovery (DR) measurement after a thermally induced concentration gradient and dynamic light scattering (DLS). For the DR experiments, the suspension is confined in a flat glass cell with a gap of $5 \mu \mathrm{m}$. A tightly focused infrared laser beam $(2 \mathrm{~W}, \lambda=1064 \mathrm{~nm})$ is used to heat a small volume, from which particles diffuse outwards due to thermodiffusion [27, 22]. The depletion region has an approximate Gaussian concentration profile. The laser is then switched off, and the dynamics of recovery of the concentration distribution $c(\vec{r}, t)$ is probed by measuring the optical transmission $T=\ln \left(I / I_{0}\right)$ of the cell, where $I=I(x, y)$ is the intensity transmitted and $I_{0}$ is the intensity value for the empty cell filled with dodecane.

The diameter of the illuminated volume is much larger than the gap width of the sample cell, so that the concentration profiles are quasi-2D. The concentration depends only on the $x$ - and $y$-coordinates, perpendicular to the propagation direction of the beam. The initial concentration profile can approximated by a Gaussian distribution,

$$
c(\mathbf{r}, t=0)=c_{0}\left[1-\frac{\Delta c}{c_{0}} \exp \left\{-\frac{r^{2}}{2 \sigma^{2}}\right\}\right],
$$

where $r=\sqrt{x^{2}+y^{2}}$ is the radial distance to the cylindrical symmetry axis of the illuminated region, and $\sigma$ is the width of the illuminated region. Furthermore, $c_{0}$ is the uniform initial concentration of particles, $\Delta c=c_{0}-c_{\text {mid }}(t=0)$, and $c_{\text {mid }}(t=0)$ is the initial concentration at the center of the illuminated volume. Assuming an isotropic diffusive dynamics of the particles with the diffusion constant $D$, we seek the solution of the diffusion equation

$$
\frac{\partial c(r, t)}{\partial t}=\underset{3}{D \nabla^{2} c(r, t)}
$$


in the form

$$
c(r, t)=\int d x^{\prime} \int d y^{\prime} G\left(\mathbf{r}-\mathbf{r}^{\prime}, t\right) c\left(\mathbf{r}^{\prime}, t=0\right),
$$

where

$$
G(\mathbf{r}, t)=\frac{1}{4 \pi D t} \exp \left(-\frac{r^{2}}{4 D t}\right),
$$

is the fundamental solution of the diffusion equation in two dimensions. Evaluating the Gaussian integrals in Eq. (3) leads to

$$
c(\mathbf{r}, t)=c_{0}\left[1-\frac{\Delta c}{c_{0}} \frac{2 \sigma^{2}}{2 \sigma^{2}+4 D t} \exp \left\{-\frac{r^{2}}{2 \sigma^{2}+4 D t}\right\}\right] .
$$

The concentration $c_{\text {mid }}(t)$ at the center-line of the illuminated volume $(r=0)$ is thus equal to

$$
c_{\text {mid }}(t)=c_{0}-\left[c_{0}-c_{\text {mid }}(t=0)\right] \frac{2 \sigma^{2}}{2 \sigma^{2}+4 D t} .
$$

We introduce a specific area

$$
s(t) \equiv \frac{\sigma^{2}}{2}\left[\frac{\Delta c}{c_{0}-c_{\mathrm{mid}}(t)}-1\right]=D t,
$$

where $\Delta c=c_{0}-c_{\text {mid }}(t=0)$. Eq. (7) shows that $s(t)$ is a linear function of time, with a slope proportional to the diffusion constant $D$. Any deviations from the linear characteristics reflect a time dependence of $D$.

The second technique that we use is dynamic light scattering (DLS). Such experiments provide an accurate and well established approach to investigate the dynamics of complex fluids. DLS has been extensively used to study dynamics of macromolecules, polymer solutions (see, e.g. [28]) and rod-like colloids (e.g. [29, 30]). DLS measures the normalized auto-correlation function $\hat{g}_{2}$ of the scattered light intensity $I(t): \hat{g}_{2}(q, t)=\langle I(t=0) I(t)\rangle /\langle I(t=0)\rangle^{2}$, where the brackets indicate time averaging over many independent realizations of positions and orientations of the colloidal particles. The parameter $q$ is the scattering wave vector between the incident and the scattered beam. For an ideal experimental setup, the normalized intensity auto-correlation function decays from two at $t=0$ to 1 for $t \rightarrow \infty$. This correlation function is related to the normalised correlation function $\hat{g}_{1}(t)$ of the scattered electric field strength $E(t)$, which is defined as $\hat{g}_{1}(q, t)=\langle E(t=0) E(t)\rangle /\langle I(t=0)\rangle$. This function is unity at $t=0$. For a sufficiently large number of scatterers within the scattering volume (such that the scattered electric field strength is a Gaussian variable), these two correlation function are related by the so-called Siegert relation [31]:

$$
\hat{g}_{2}(q, t)=1+\hat{g}_{1}^{2}(q, t) .
$$

For optically anisotropic particles, two types of DLS experiments can be performed, where the incident light is polarised perpendicular to the plane spanned by the directions of the incident and scattered beam, but where the detected polarisation direction is either in the same plane, or within the plane. These two experiments are referred as VV and VH experiments (with V for vertical and $\mathrm{H}$ for horizontal), respectively. Translational diffusion coefficients can be obtained from VV experiments, while the rotational diffusion coefficient can be probed with VH experiments. 
For monodisperse particles at low concentrations, where neither interactions of particles nor multiple scattering play a role, the correlation functions obtained by DLS experiments are single exponentials in time [31],

$$
\hat{g}_{1}(t)=A \exp (-\Gamma t)
$$

where $\Gamma$ is the decay rate. The constant $A$ accounts for mixing of different scattering vectors probed due to the finite extent of the scattering volume. This renders the amplitude $A$ typically of the order of 0.95 . In VV experiments, the decay rate is related to the mean diffusion coefficient $D$ as [31],

$$
\Gamma_{\mathrm{VV}}=D q^{2}
$$

provided that the degree of anisotropy is small (typically the relative differences in dielectric constants of the particles should be of the order of, or less than, 0.1). For the particles in the present study, this requirement is fulfilled. For VH experiments, there is a contribution to the decay rate independent of the scattering wave vector, even for small optical anisotropies, which is proportional to the rotational diffusion coefficient $D_{\mathrm{r}}[31]$,

$$
\Gamma_{\mathrm{VH}}=D q^{2}+6 D_{\mathrm{r}}
$$

Polydispersity gives rise to deviations from this dependence, which can be accounted for in several ways. One solution is the so-called cumulant method [32], where the exponent in Eq. (9) is expanded in a power series of the time $t$ :

$$
\hat{g}_{1}(q, t)=A \exp \left(-\bar{\Gamma} t+\Gamma_{2} t^{2}+\ldots\right)
$$

This expression is valid for short times, that is, it describes the initial decay of the correlation function [33]. The first coefficient yields the mean diffusion coefficient, as given in Eqs. 10 and 11, while the second one describes the distribution of the relaxation rates. In most cases (for relatively small polydispersity), it is sufficient to consider only terms up to second order. An alternative method, which is also valid for long times and arbitrary polydispersity, is to approximate the correlation function by a sum of several relaxation rates,

$$
\hat{g}_{1}(q, t)=\sum_{j} g_{j} \exp \left(-\Gamma_{j} t\right),
$$

where $g_{j}$ is the amplitude of the mode with the decay rate $\Gamma_{j}$. Distributions of amplitudes can be determined from experimental correlation functions through an inverse Laplace transform technique [34]. CONTIN is a standard program that implements this technique using a regularization procedure.

Dynamic light scattering experiments have been performed here both in VV and VH geometries. Two different setups were used for low concentrations (less than $<0.5 \mathrm{wt} \%$ ), which only differed in the correlator types and the wavelengths of the laser (647 and $632 \mathrm{~nm}$, resp.). After passing the analyzer, the scattered light was led through a photomultiplier coupled to a correlator. Different scattering angles were achieved by mounting the analyser and the photomultiplier on a goniometer. In both setups, the sample was placed in a toluene bath to avoid scattering from imperfections of the glass cuvette. The scattering angle was varied between $30^{\circ}$ and $150^{\circ}$. For concentrations of $0.5 \mathrm{wt} \%$ and higher, another setup was used: the sample was confined between a pair of flat planar glass plates (with gap widths of $10 \mu \mathrm{m}$ to $50 \mu \mathrm{m}$ ) to minimise multiple scattering. The scattering angle could be varied in that setup between $5^{\circ}$ and $60^{\circ}$. The relaxation 
rates $\Gamma$ were extracted from the fits of the corresponding intensity correlation functions $C(t)$ for different scattering vectors $q$. The diffusion coefficients we obtained from the fits of $\Gamma(q)$ using equations (10) and (11), respectively.

\section{Results}

\subsection{Thermo-Depletion Recovery Experiments}

Figure 2 shows the specific area $s(t)$, as defined in Eq. (7), for different concentrations $c_{0}$ of the suspension. All curves are averaged over several measurements at the same experimental conditions. For concentrations $c<25 \mathrm{wt} \%$, the specific area is a linear function of time, as expected from Eq. (7). Only at $c=25 \mathrm{wt} \%$, deviations from the linear dependence are observed. Due to the concentration dependence of the diffusion coefficient $D$ in this region, the diffusion equation (2) can only be applied in the final stage of depletion recovery, when the local concentration within the depletion region is close to the ambient concentration $c_{0}$.
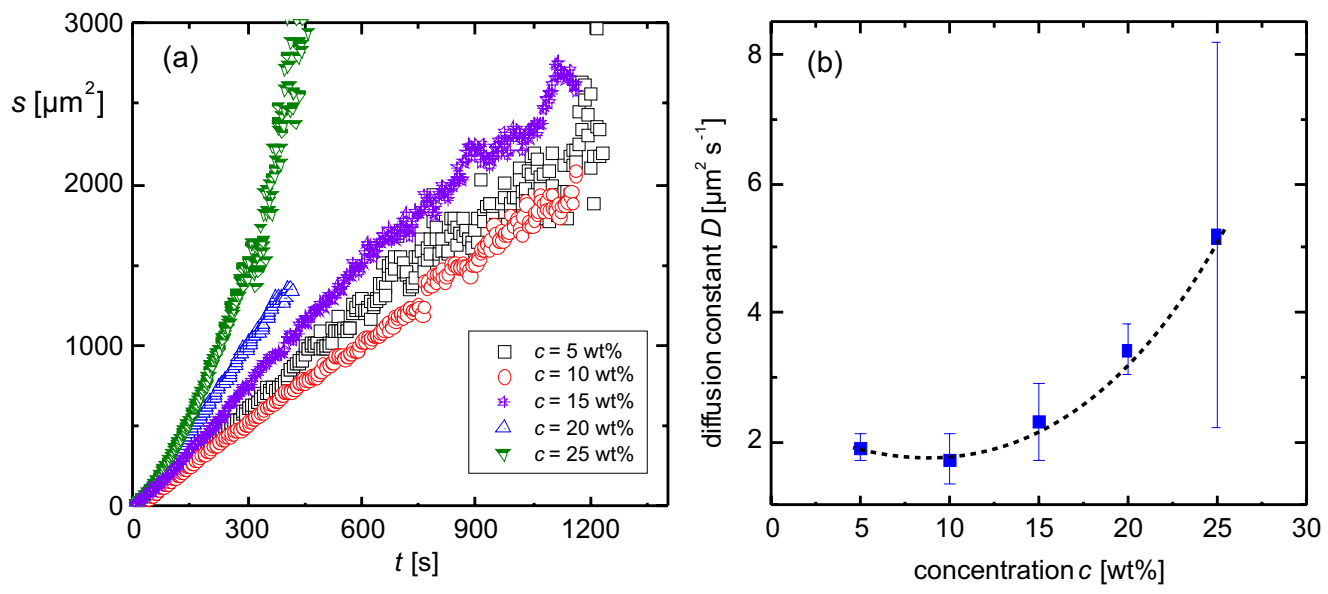

Figure 2: a) Specific area $s(t)$ as a function of time for different concentrations. b) Diffusion constants determined from the time dependence of the specific area. The dotted line guides the eye.

The obtained diffusion constants are shown in Fig. 2b. In view of the above discussed reason for the non-linear behaviour of $s(t)$ for the highest concentration, the diffusion coefficient for that concentration is obtained from the final slope of the parameter $s(t)$ in Fig. 2a at large times. The diffusion constant strongly increases with concentration, which is to be expected for particles with a repulsive interaction potential.

\subsection{DLS Experiments}

Correlation functions for the concentration $\mathrm{c}=0.1 \mathrm{wt} \%$ are exemplarily shown in Fig. 3. It is obvious that the data cannot be described well by a single exponential decay (see Eq. (9)). The deviations are particularly strong for small scattering angles in VH geometry.

The reason for this is most likely the large polydispersity and the interparticle interactions. To account for polydispersity, both CONTIN and the cumulant methods were used. The relaxation rate distribution function was determined by setting the regularisation parameter to $\alpha=1$, as 

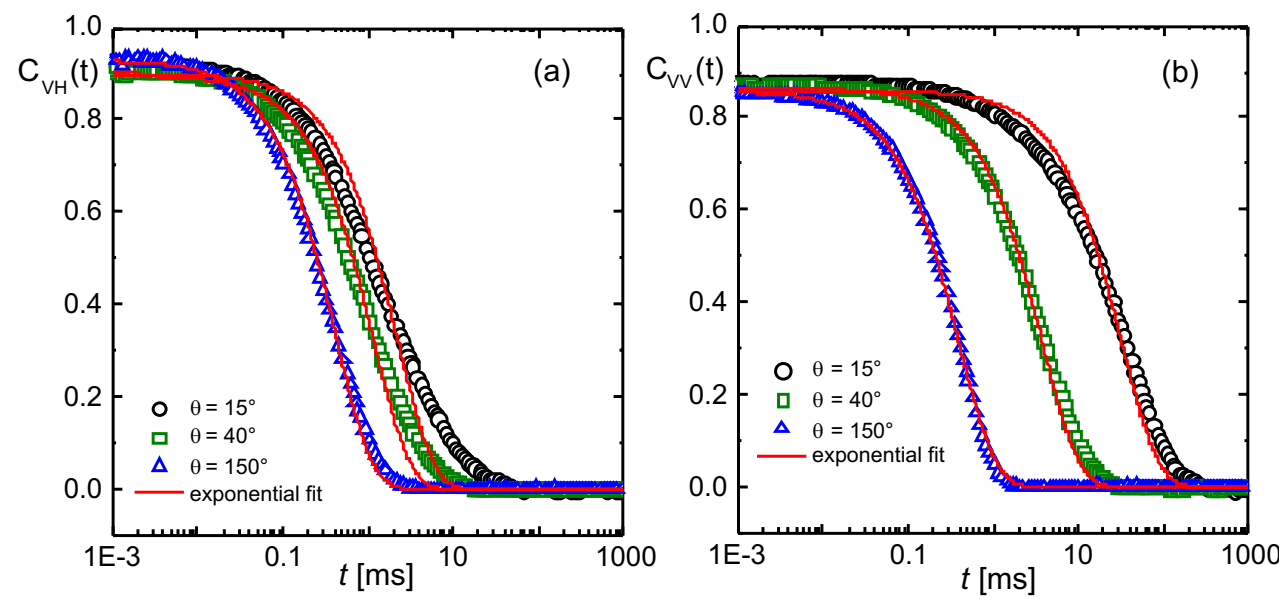

Figure 3: Examples of DLS intensity correlation functions for a concentration $c=0.1 \mathrm{wt} \%$ a) VH and b) VV. The solid red lines are best fits to a single exponent. Clearly, the correlation function do not follow a single exponential decay.

a good compromise between a satisfactory peak resolution of the distribution function and a reasonable smoothness. Some examples of the distribution functions are shown in Figs. $4 \mathrm{a}$ and $4 \mathrm{~b}$, where the distribution functions were normalised to 1 at the most probable relaxation rate.

Because of the relatively large polydispersity, the cumulant method can only be used for the initial decay of correlation functions. The cut-off time is chosen as the largest time where a second-cumulant fit (see Eq. (12)) still describes the data accurately.

From the relaxation rates determined by the two methods at different angles, the dispersion relations for $\Gamma$ can be obtained (see Figs. 6 and 7). As expected, the relaxation rate depends almost linearly on the square of the wave number $q$ (see Eqs. (10) and (11)). The VH dispersion curve has an offset related to the rotational diffusion constant. The relatively large scattering of the relaxation rates is due to stability problems of the CONTIN routine, because the determined distribution function is very sensitive to noise in the correlation function and also to the chosen range of relaxation rates. The choice of the regularisation parameter also influences the result. The dispersion relations from the cumulant analysis for low concentrations $(c<0.1 \mathrm{wt} \%)$ look similar to that of the CONTIN results. At high concentrations, however, the VV dispersion relations have an offset of about $100 \mathrm{~s}^{-1}$. A possible explanation for this is multiple scattering, which may influence the correlation function.

These dispersion relations, examples of which will be shown later, allow us to determine the translational and rotational diffusion constants, $D_{t}$ and $D_{r}$. The translational diffusion constant $D_{t}$ was determined from the slope of the VV dispersion relations, while the rotational diffusion constant was determined from the offset of the VH dispersion relation. Figure 8 shows the diffusion constants, where the values determined from the CONTIN analysis are given as black squares and the values from the cumulant analysis red dots. The influence of the concentration on the translational diffusion constant is relatively small, taking into account the relatively large experimental error. $D_{t}$ grows slightly with increasing concentration in the range of low concentrations $(<0.5 \%)$, reaching a maximum at about $0.5 \mathrm{wt} \%$ and then, it decreases at higher concentrations. The rotational diffusion constant shows a similar behaviour, but, in this case, an increase of the 

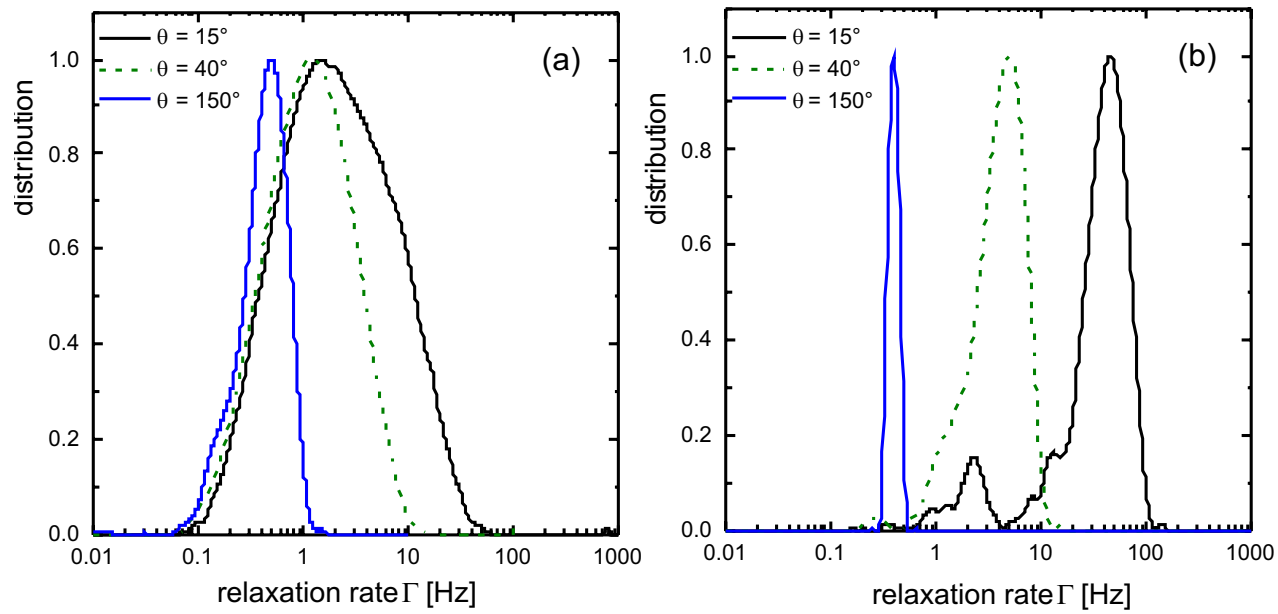

Figure 4: Examples of distribution functions from the CONTIN analysis for a concentration $c=0.1 \mathrm{wt} \%$, a) $\mathrm{VH}$ and b) VV
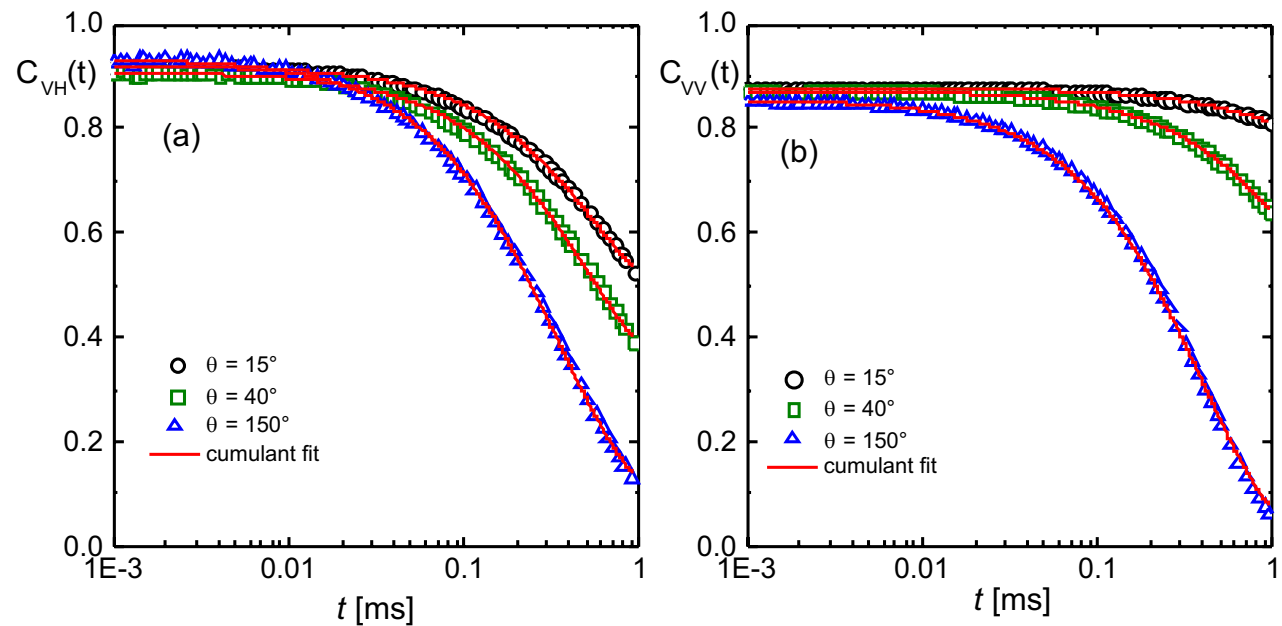

Figure 5: Examples of cumulant fit functions for a concentration $c=0.1 \mathrm{wt} \%$, a) VH and b) VV 

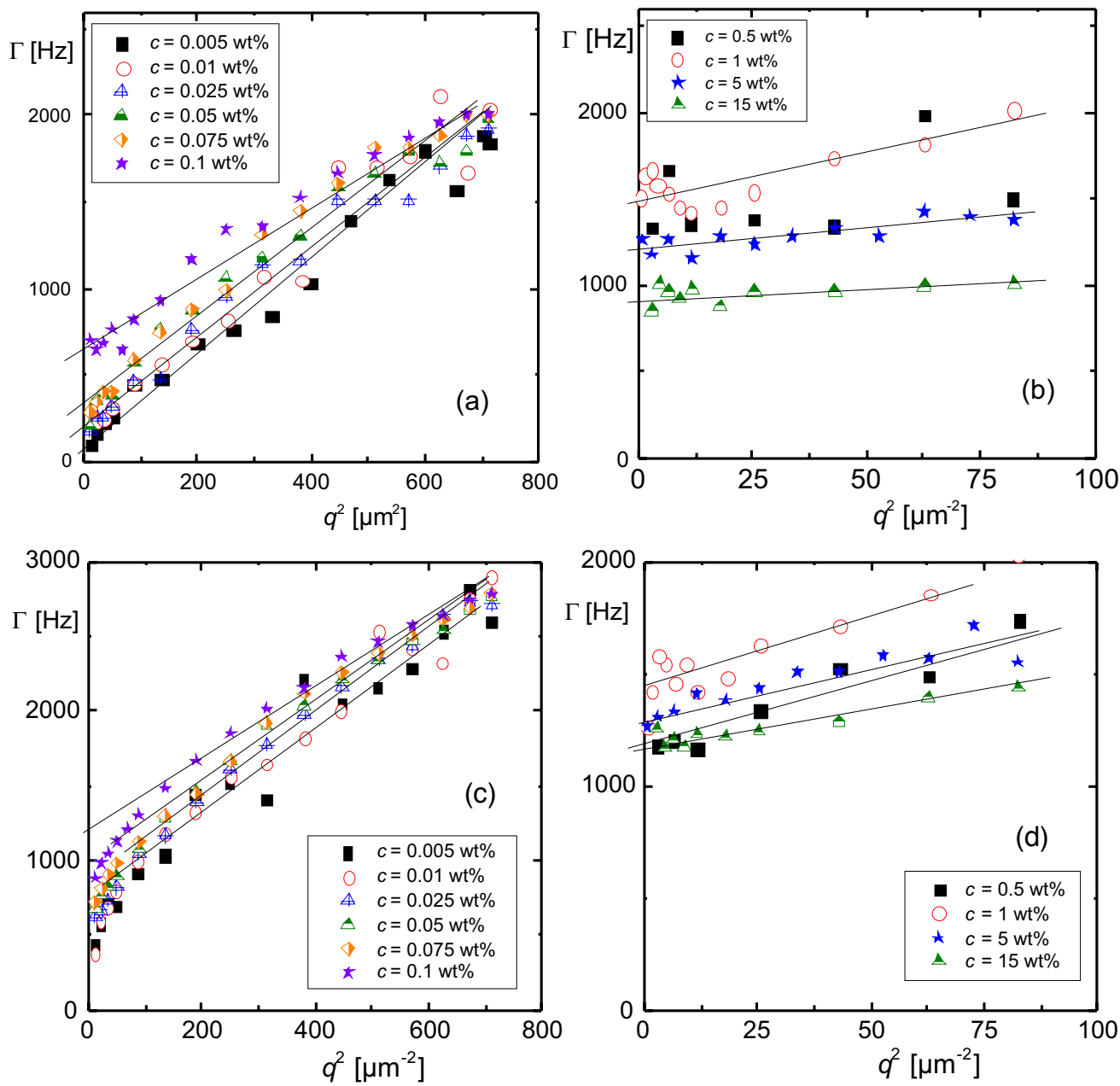

Figure 6: VH dispersion relations from CONTIN analysis, a) low and b) high concentrations, and from cumulant analysis, c) low and d) high concentrations 

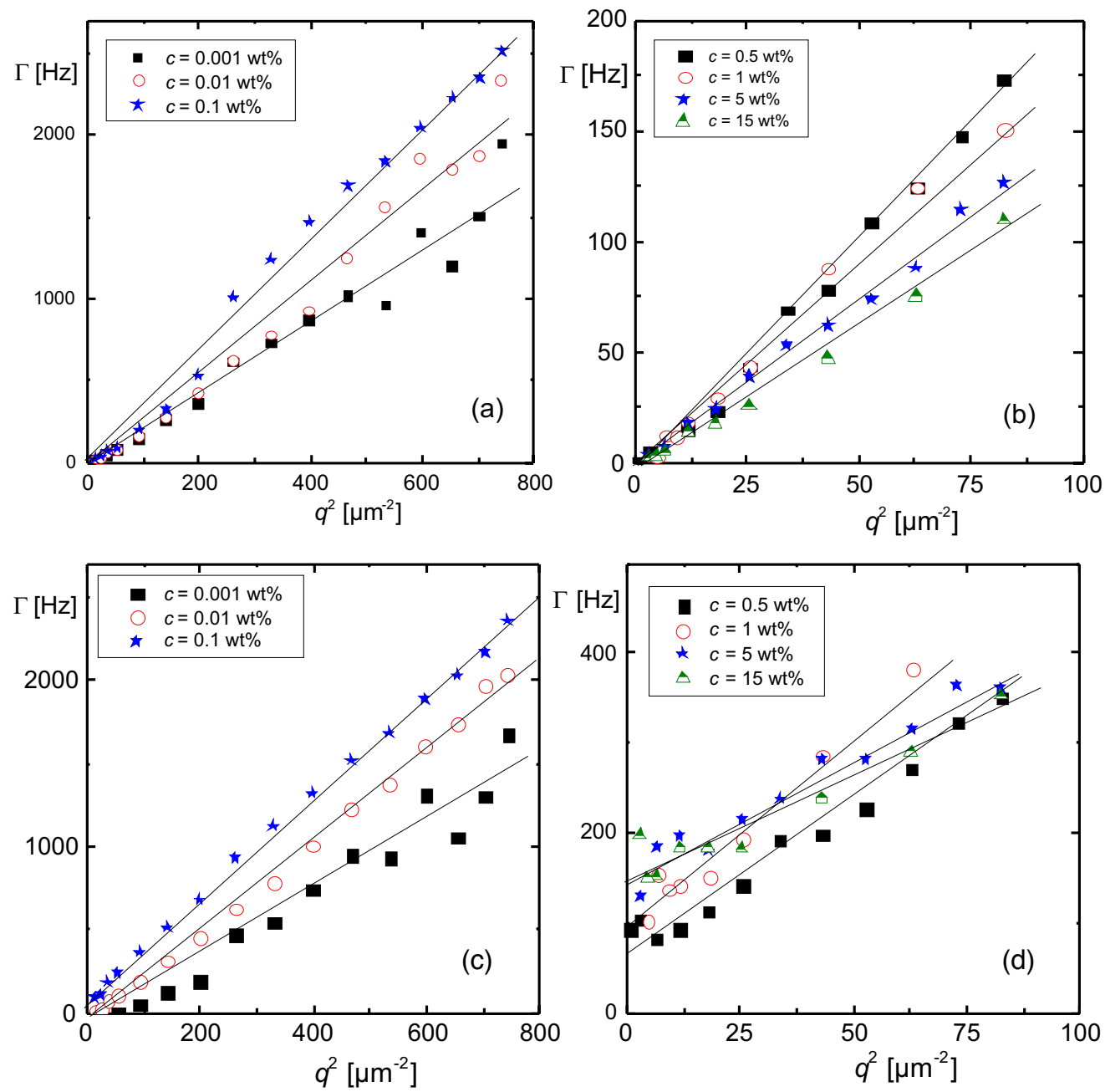

Figure 7: VV dispersion relations from CONTIN analysis, a) low and b) high concentrations, and from cumulant analysis, c) low and d) high concentrations 
concentration by a factor 100 leads to an increase of the rotational diffusion constant by a factor of about 3 to 10, depending on the analysis method. The diffusion constants for both methods of analysis show the same trend, but the error ranges do not intercept in most of the cases.
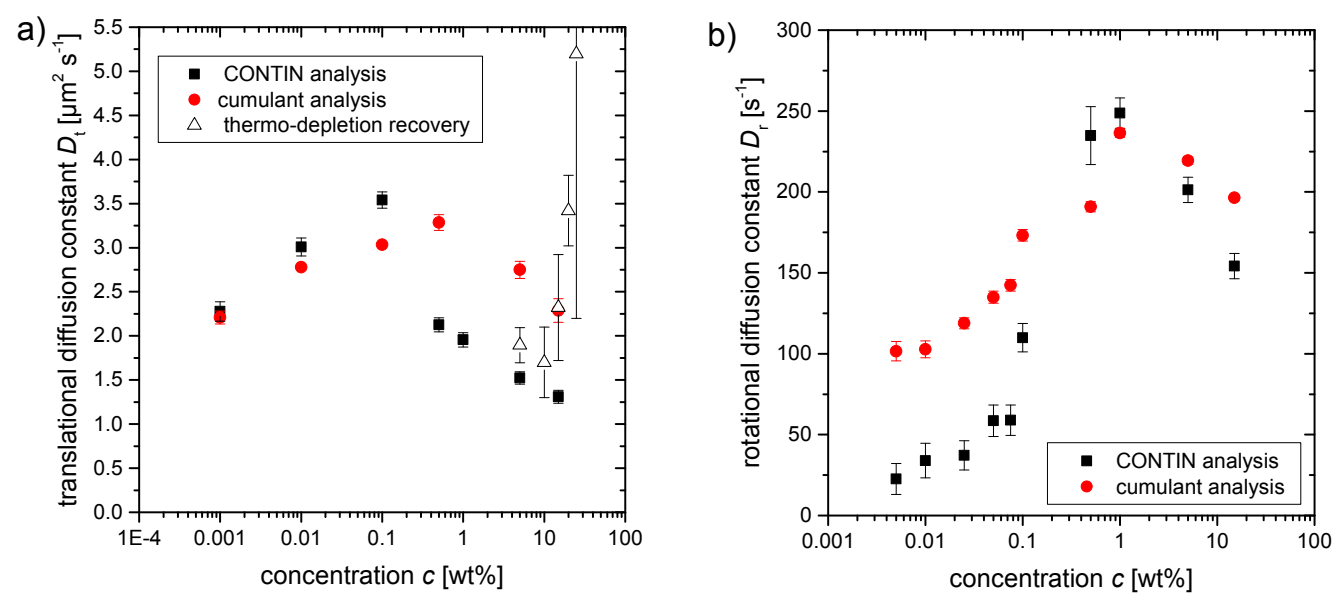

Figure 8: Diffusion coefficients in dependence of suspension concentration measured by different techniques, a) translational diffusion and $b$ ) rotational diffusion

\section{Discussion}

Microscopic free-diffusion constants of non-interacting rods suspended in an isotropic fluid with the viscosity $\eta$ can be estimated using the theory of diffusion of rod-shaped particles $[35,36]$

$$
D_{\|}=\frac{k_{b} T}{2 \pi \eta L}\left(\ln \frac{L}{D}+\gamma_{\|}\right), D_{\perp}=\frac{k_{b} T}{4 \pi \eta L}\left(\ln \frac{L}{D}+\gamma_{\perp}\right)
$$

where $\gamma_{\|}$and $\gamma_{\perp}$ are constants determined by the aspect ratios of the rods. On a long-time scale, the motion of the particles in random directions can be described by the macroscopic (mean) diffusion constant

$$
\bar{D}=\frac{k_{b} T}{3 \pi \eta L}\left(\ln \frac{L}{D}+\gamma\right)
$$

Substituting the values for the viscosity of dodecane and the parameters of the particles, $\gamma_{\|}=-0.0163, \gamma_{\perp}=0.8853$ and,$\gamma=0.4210$ [35], theory predicts a coefficient for diffusion along the particle axis $D_{\|}=(3.3 \pm 1.5) \mu \mathrm{m}^{2} \mathrm{~s}^{-1}$ and perpendicular to the axis $D_{\perp}=(2.6 \pm 1.5)$ $\mu \mathrm{m}^{2} \mathrm{~s}^{-1}$ with a mean of $\bar{D}=(2.8 \pm 1.5) \mu \mathrm{m}^{2} \mathrm{~s}^{-1}$ at $T=293 \mathrm{~K}$.

The experimental results show, that the collective diffusion coefficient grows with increasing concentration in the concentration range $5-25 \mathrm{wt} \%$, see Figure $2 \mathrm{~b}$. This is a surprising effect, because with increasing concentration the viscosity of the suspension increases, and a decrease of the collective diffusion constant would be expected. There are two possible mechanisms which could lead to an increased collective diffusion at high concentrations: interactions (hydrodynamic and long-range) or alignment of the rod-shaped particles. The influence of interactions on the 
diffusion of colloids has been shown by Anderson et al. [37, 38]. They found an increase of the diffusion coefficient with concentration of the colloid, depending on its ionic strength. A similar effect has been reported by Zahn et al. [39], who found an increase of the 2D self-diffusion coefficient with concentration due to hydrodynamic interactions. Another potential reason is the alignment of the particles. In thermotropic nematic liquid crystals, the diffusion constant along the local director usually is faster than perpendicular to the director [40].

The experimental results from the light scattering experiments show a more complex behaviour, see Figure 8 . The values for the translational diffusion coefficients satisfactory agree with mean predicted values for free-particle diffusion. The rotational diffusion constants are lower then the predicted values, between $245 s^{-1}$ and $270 s^{-1}$, in the experiment, suggesting a significant hindrance of particle rotation. The deviations of the rotational dynamics from the theory may also be accounted by the polydispersity of the pigment particles [35]. In the experiments of Tirado et al., the dynamics slow down at high concentrations. This can be explained by the increased viscosity of the suspension surrounding a single particle and the steric hindrance. This is in good agreement with the findings in other colloidal systems: in contrast to collective diffusion with interparticle interactions, the self diffusion coefficient of colloidal rods has been found to decrease with concentration [41]. Van Bruggen et al. also found a strong decrease (by a factor of about 10) of $D_{\|}$and $D_{\perp}$ at the transition to the nematic state. The explanation for this is the enhanced density in the nematic phase due to a closer packing. So in the case of our DLS experiments with pigment rods at concentrations above $1 \mathrm{wt} \%$, the influence of the increased density seems to be stronger than the influence of the interactions. In contrast to that, at concentrations lower than $1 \mathrm{wt} \%$, density differences do not seem to be important, while interactions still play a role. Qualitatively, there is no difference between the short-time diffusion, determined by the cumulant method, and the long-term diffusion, determined by the CONTIN analysis. In general, the long-term dynamics are slower than the short-term dynamics. This is expected, because the interactions hinder free diffusion. Especially at high concentrations, where interactions are more frequent, slower dynamics in the long-term analysis are expected. The translational diffusion constant shows exactly this, Figure $8 \mathrm{a}$. The rotational dynamics is slowed down by the interactions already at low concentrations (Figure 8b).

\section{Summary}

The collective dynamics of anisometric pigment particles was investigated. Both translational and rotational diffusion coefficients exhibit complex dependences on the concentration of pigment particles. $D_{\mathrm{r}}$ slightly increases in the isotropic range of concentration and exhibits a strong increase near the transition into the orientationally ordered state. The rotational diffusion coefficient exhibits a maximum in the isotropic range and decreases in the anisotropic one. It appears that the orientational correlations of the particles enhance the diffusion despite the competing effect of the density increase.

\section{Acknowledgments}

Financial support from the German Science Foundation (DFG) via the priority programme SPP 1681 is gratefully acknowledged and DAAD.

[1] J. N. Israelachvili, Intermolecular And Surface Forces: With Applications to Colloidal and Biological Systems, Academic Press, London, 1992. 
[2] S. A. Safran, Statistical Thermodynamics Of Surfaces, Interfaces, And Membranes, Addison-Wesley, Weizmann Institute of Science, Rehovot, Israel, 1994.

[3] P. G. de Gennes, J. Prost, The Physics of Liquid Crystals, Clarendon Press, 1995.

[4] S. Fraden, Phase Transitions in Colloidal Suspensions of Virus Particles, Nato Science Ser. C, 1995, pp. $113-164$.

[5] S. Fraden, G. Maret, D. L. Caspar, R. B. Meyer, Isotropic-nematic phase transition and angular correlations in isotropic suspensions of tobacco mosaic virus., Phys. Rev. Lett. 63 (1989) 2068-2071.

[6] S. Fraden, A. Hurd, R. B. Meyer, Electric-field-induced association of colloidal particles, Phys. Rev. Lett. 63 (1989) 2373-2376.

[7] Z. Dogic, A. P. Philipse, S. Fraden, J. K. G. Dhont, Concentration-dependent sedimentation of colloidal rods, J. Chem. Phys. 113 (2000) 8368-8380.

[8] Z. Dogic, S. Fraden, Cholesteric phase in virus suspensions, Langmuir 16 (2000) 7820-7824.

[9] Z. Dogic, D. Frenkel, S. Fraden, Enhanced stability of layered phases in parallel hard spherocylinders due to addition of hard spheres, Phys. Rev. E 62 (2000) 3925-3933.

[10] Z. Dogic, S. Fraden, Smectic phase in a colloidal suspension of semiflexible virus particles, Phys. Rev. Lett. 78 (1997) 2417-2420.

[11] Z. X. Zhang, J. S. van Duijneveldt, Isotropic-nematic phase transition of nonaqueous suspensions of natural clay rods, J. Chem. Phys. 124 (2006) 154910.

[12] E. van den Pol, A. V. Petukhov, D. M. E. Thies-Weesie, G. J. Vroege, Simple rectangular columnar phase of goethite nanorods and its martensitic transition to the centered rectangular columnar phase, Langmuir 26 (2010) 1579-1582.

[13] R. G. Larson, The structure and rheology of complex fluids, Oxford University Press, 1999.

[14] P. L. Reece, Smart Materials and Structures, New Research, Nova Publishers, 2007.

[15] N. Yasarawan, J. S. van Duijneveldt, Dichroism in dye-doped colloidal liquid crystals, Langmuir 24 (2008) 7184 7192.

[16] R. J. Greasty, R. M. Richardson, S. Klein, D. Cherns, M. R. Thomas, C. Pizzey, N. Terrill, C. Rochas, Electroinduced orientational ordering of anisotropic pigment nanoparticles, Phil. Trans. R. Soc. Lond. A 371 (2013) 20120257.

[17] A. Eremin, R. Stannarius, S. Klein, J. Heuer, R. M. Richardson, Switching of electrically responsive, light-sensitive colloidal suspensions of anisotropic pigment particles, Adv. Funct. Mater. 21 (2011) 556-564.

[18] E. Grelet, M. Paul Lettinga, M. Bier, R. van Roij, P. van der Schoot, Dynamical and structural insights into the smectic phase of rod-like particles, J Phys-Condens Mat 20 (49) (2008) 494213.

[19] K. Kang, Glass transition of repulsive charged rods (fd-viruses), Soft Matter 10 (18) (2014) 3311-14.

[20] K. Kang, J. K. G. Dhont, Structural arrest and texture dynamics in suspensions of charged colloidal rods, Soft Matter 9 (17) (2013) 4401-12.

[21] K. K, J. K. G. Dhont, Glass Transition in Suspensions of Charged Rods: Structural Arrest and Texture Dynamics, Phys. Rev. Lett. 110 (1) (2013) 015901-4.

[22] K. May, R. Stannarius, S. Klein, A. Eremin, Electric-field-induced phase separation and homogenization dynamics in colloidal suspensions of dichroic rod-shaped pigment particles, Langmuir 30 (2014) 7070-7076.

[23] R. Greasty, J. Heuer, S. Klein, C. Pizzey, R. Richardson, Electric field induced orientational order in suspensions of anisotropic nanoparticles, Mol. Cryst. Liq. Cryst. 545 (2011) 133-145.

[24] M. U. Schmidt, D. W. M. Hofmann, C. Buchsbaum, H. J. Metz, Crystal Structures of Pigment Red 170 and Derivatives, as Determined by X-ray Powder Diffraction, Angew. Chem. Int. Edit. 45 (2006) 1313-1317.

[25] F. Campbell, P. Davies, J. Schofield, Dispersion, US patent 4,645,611.

[26] R. Greasty, Orientational order in pigment particle suspensions, Ph.D. thesis, University of Bristol (2012).

[27] S. Duhr, D. Braun, Why molecules move along a temperature gradient, P Natl Acad Sci Usa 103 (52) (2006) 19678-19682.

[28] R. Pecora, Doppler shifts in light scattering from pure liquids and polymer solutions, J. Chem. Phys. 40 (1964) 1604-1614.

[29] S. De Sio, P. R. Lang, Depletion interaction mediated by fd-virus: on the limit of low density and Derjaguin approximation, Z. Phys. Chem. 229 (2014) 1161-1175.

[30] B. M. I. van der Zande, J. K. G. Dhont, M. R. Böhmer, A. P. Philipse, Colloidal dispersions of gold rods characterized by dynamic light scattering and electrophoresis, Langmuir 16 (2000) 459-464.

[31] B. J. Berne, R. Pecora, Dynamic Light Scattering: With Applications to Chemistry, Biology, and Physics, Dover, New York, 2000.

[32] D. E. Koppel, Analysis of macromolecular polydispersity in intensity correlation spectroscopy: The method of cumulants, J. Chem. Phys. 57 (1972) 4814-4820.

[33] P. A. Hassan, S. K. Kulshreshtha, Modification to the cumulant analysis of polydispersity in quasielastic light scattering data, J. Colloid Interface Sci. 300 (2006) 744-748.

[34] S. W. Provencher, A constrained regularization method for inverting data represented by linear algebraic or integral 
equations, Computer Physics Communications 27 (1982) 213-227.

[35] M. M. Tirado, C. L. Martínez, J. G. de la Torre, Comparison of theories for the translational and rotational diffusion coefficients of rod-like macromolecules. application to short dna fragments, J. Chem. Phys. 81 (1984) 2047-2052.

[36] F. C. Cheong, D. G. Grier, Rotational and translational diffusion of copper oxide nanorods measured with holographic video microscopy, Optics Express 18 (2010) 6555-6562.

[37] J. L. Anderson, C. C. Reed, Diffusion of spherical macromolecules at finite concentration, J. Chem. Phys. 64 (1976) $3240-3250$.

[38] J. L. Anderson, F. Rauh, A. Morales, Particle diffusion as a function of concentration and ionic strength, J. Phys Chem. 82 (1978) 608-616.

[39] K. Zahn, J. M. Méndez-Alcaraz, G. Maret, Hydrodynamic interactions may enhance the self-diffusion of colloidal particles, Phys. Rev. Lett. 79 (1997) 175-178.

[40] S. V. Dvinskikh, I. Furó, Anisotropic self-diffusion in the nematic phase of a thermotropic liquid crystal by ${ }^{1} \mathrm{H}-$ spin-echo nuclear magnetic resonance, J. Chem. Phys. 115 (2001) 1946-1950.

[41] M. P. B. van Bruggen, H. N. W. Lekkerkerker, G. Maret, J. K. G. Dhont, Long-time translational self-diffusion in isotropic and nematic dispersions of colloidal rods, Phys. Rev. E 58 (1998) 7668-7677. 